\title{
El marketing en las pymes
}

Marketing in SMEs

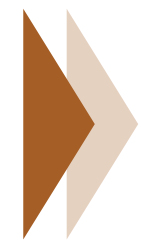

Martha Cecilia Pachón

Administradora de Empresas Comerciales - Universidad Colegio Mayor de Cundinamarca, Especialista en Formulación y Evaluación Social y Económica de Proyectos - Universidad Católica (Colombia), Mg. en Mercadeo, Universidad Externado de Colombia. Docente

Pregrado y Postgrado - Facultad de Ciencia y Tecnologías, VUAD, Universidad Santo Tomas Sede Bogotá.

E-mail: marthapachon@ustadistancia.edu.co

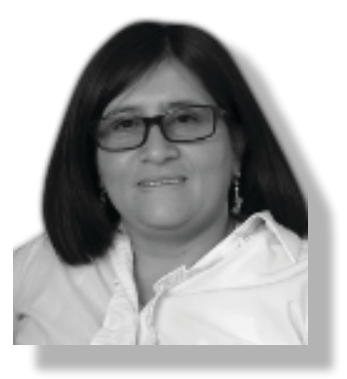




\section{RESUMEN}

Este artículo rescata algunos de los aportes efectuados por algunos autores, quienes, gracias a estudios e investigaciones, han llegado a la conclusión de la importancia que tiene la gestión comercial dentro de las pymes. En este sentido, las pymes ubicadas en la ciudad de Bogotá, Colombia, no son ajenas al tema, toda vez que requieren de elementos que les permita dar a conocer sus productos y servicios, generando valor agregado, así como el fortalecimiento la relación con sus clientes; razón por la cual el uso del Marketing cobra más fuerza en las organizaciones, al ser una herramienta necesaria para que las estas sean más competitivas, sin importar su tamaño.

Palabras Claves: Marketing, redes sociales, pymes, web, competitividad.

\section{ABSTRACT}

This article rescues some of the contributions made by authors who, thanks to studies and research, have concluded the importance of business management within SMEs. In this regard, SMEs located in Bogota, Colombia, are not unrelated to the subject, since they require elements that allow them to publicize their products and services, generating added value and strengthen the relationship with their customers; why use Marketing is stronger in organizations, being a necessary condition for organizations to be more competitive, regardless of the size of these tool.

Keywords: Marketing, social networks, SMEs, web, competitiveness.

\section{Introducción}

En el escenario actual donde confluyen las empresas, independiente de su tamaño, cada vez se hace más necesario el fortalecimiento de las relaciones con el cliente, y en muchas ocasiones esto se da gracias a poder contar con herramientas TIC, como las redes sociales y el Internet, las cuales se han convertido en una herramienta para los clientes y usuarios de un producto o servicio, a la hora comprar, porque les permite acceder a la información en tiempo real, ver las opiniones de otros clientes o usuarios, hasta comparar varias opciones para optar por la que más les beneficia o la que mejor convence.

Hablar de marketing aplicado en las pymes es un tema muy extenso, más sí se tiene en cuenta los tipos de marketing y su aplicabilidad dentro de las organizaciones. En este artículo se pretende rescatar la importancia del concepto y las acciones que implica llevar a cabo un excelente marketing como estrategia para fortalecer la gestión comercial, estrechar relaciones con los clientes y fortalecimiento de las políticas de fidelización.

"El marketing no es sinónimo de comercialización o venta, sino que es, precisamente, la función que ayuda a identificar esas necesidades desarrollando los productos para satisfacerlas, colaborando a fijar los precios correspondientes, ofreciéndolos en los lugares adecuados y con buenas estrategias de comunicación y postventa. El marketing orienta todo el proceso mediante el cual las ideas se transforman en productos aceptados por el mercado. Por eso la importancia del marketing en Pymes resulta cada vez más evidente". (Schnarch, 2013)

Hoy en día en un país como Colombia, donde desde la labor de relaciones a nivel internacional, se establecen convenios comerciales, donde no sólo se trabaja en la gestión de exportación de productos, bienes y servicios, sino que se establecen acuerdos para fortalecer las importaciones; donde al final del ejercicio se benefician claramente los consumidores, porque tienen varias opciones a la hora de efectuar una compra; pero para las pymes colombianas que no se adaptan con facilidad a los cambios, que no fortalecen el desarrollo de sus procesos, el que más empresas ingresen al mercado nacional, les genera mayor dificultad de vender sus productos o servicios, haciendo más necesario el tema de fortalecer su gestión comercial, como estrategia para ser competitivo; es decir, que no basta hablar de contar con un buen producto o servicio, se debe tener presente cómo se le va a llegar al cliente, cómo lo involucramos con lo que se está haciendo, entre otros factores que implica acercar más la empresa al cliente. 


\section{Antecedentes}

\author{
Imagen 1: Marketing y sus implicaciones. Fuente: \\ Elaboración propia Programa https://tagul.com/cloud/2
}

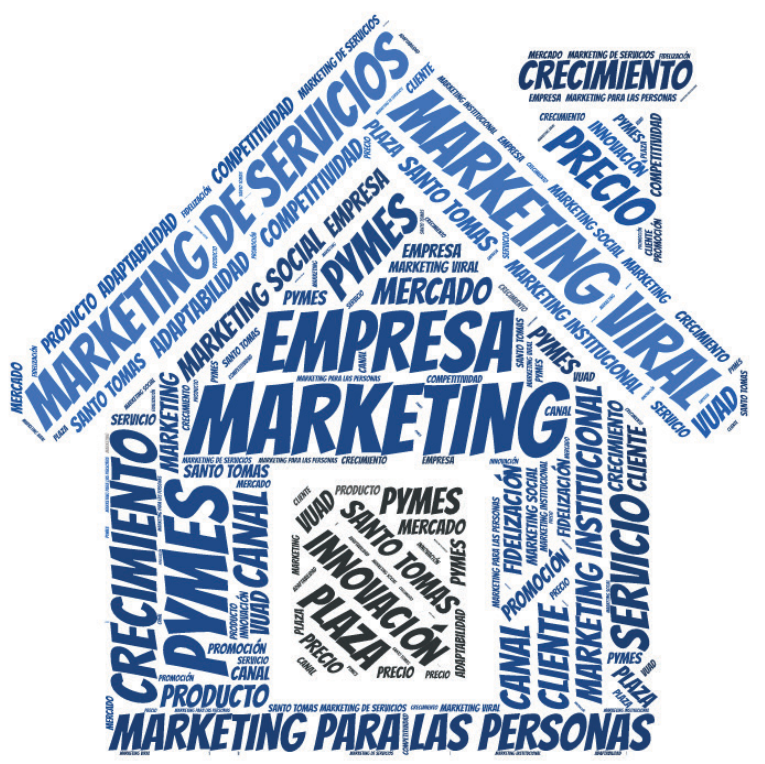

Muchas de las pymes son lideradas por personas que con mucho esfuerzo han logrado crear una empresa y con ella generar algunos empleos, y desde sus inicios les ha ido bien, lo que lleva a pensar a algunos de estos empresarios, que no son necesarios los cambios o la implementación de estrategias desde el aspecto comercial, porque como muchos de ellos afirman, "para qué más, sí así estamos bien", "para qué hacer gastos inoficiosos", "ya nos conocen, no es necesario de incurrir en más gastos”, entre otros argumentos que en ocasiones se tienen, cuando de justificar la escasa inversión en estrategias de marketing, se trata.

No se puede desconocer que hoy en día los cambios, producto de una globalización más desarrollada, cambios tecnológicos, cambios a nivel cultural, entre otros factores, han hecho que los clientes, así sean los habitantes de un barrio, estén más interesados en conocer los detalles más mínimos acerca del producto o servicio que les llama la atención, porque esperan que los mismos cumplan con sus necesidades y expectativas al ciento por ciento.
En este caso se puede hablar por ejemplo de la generación millennials, personas muy bien informadas acerca de los productos o servicios que desean comprar para satisfacer sus necesidades o gustos.

"Los millennials o la llamada "Generación Y" son los nacidos después de 1985 hasta mitad de 2000. Muchos de ellos están incorporándose al mercado laboral, y empiezan a formar parte de la población que toma decisiones a la hora de consumir e invertir. Se trata de la primera generación que ha tenido acceso a internet durante todos sus años formativos y que mayor impacto tecnológico ha recibido, por lo que su nivel de acceso a la información es más profundo y diversificado. Han experimentado toda la burbuja tecnológica" (El Tiempo, 2015).

Actualmente es normal ver a los adolescentes involucrados con las temáticas expuestas en las redes sociales, siendo los que más consultan por ejemplo en las mismas, como se puede apreciar en el siguiente aparte:

Los millennials tienen confianza en sí mismos, están conectados con el mundo y abiertos al cambio, pero lo que los mueve es la pasión. Es la generación del "aquí y ahora", de la inmediatez, resultado de haber nacido ya conectados a los canales online. El último estudio sobre las redes sociales de IAB Spain revela que el $97 \%$ de los adolescentes están presentes en las redes sociales (un $78 \%$ en Facebook, un 70\% en Youtube, $61 \%$ en Twitter y un $60 \%$ en Instagram), $y$ disponen de una infinita cantidad de información al alcance de un clic (a diferencia de la generación anterior, que necesitaba recurrir a bibliotecas o expertos). Esta sobredosis de información ha creado una generación con un mayor espiritu crítico, concienciada de los problemas sociales y medioambientales (El Tiempo, 2015).

Hoy en día es normal ver jóvenes consultando en las redes sociales opiniones frente a un tema en particular, opiniones acerca de la calidad y nivel de percepción que tienen los usuarios de determinada tecnología, del servicio ofrecido por algunas empresas, entre otros temas que son expuestos en las redes sociales. 
Así mismo, se puede ver que muchas personas adultas, están tomando la tecnología como una alternativa de consulta, transacciones vía web, con el propósito de ahorrar tiempo.

Las empresas y los empresarios pymes también deben aprender a valorar la labor de ventas y disminuir la rotación de ese personal; dejar el divorcio entre las políticas financieras, las políticas comerciales y el mercadeo; abandonar el afán por el flujo de caja y hacer seguimiento al cliente, aunque no facture.

Los empresarios pyme, hoy en día no pueden desconocer la gestión que se debe llevar a cabo desde la parte comercial, para fortalecer relaciones con sus clientes, implicando esta acción, disponer de recursos económicos y unas métricas que permitan conocer el avance de las actividades propuestas con relación a la gestión comercial que la empresa desea llevar a cabo para mejorar su nivel de competitividad.
Philip Kotler, define el proceso de marketing en cinco fases claramente identificadas: 1) investigación de mercado y entorno competitivo; 2) definición de mercado objetivo; 3) establecimiento de la estrategia de marketing; 4) gestión del marketing mix y 5) control. Estos elementos hoy en día cobran fuerza en todas las organizaciones, porque no es sólo pensar en la estrategia como una herramienta para las grandes organizaciones, se puede adaptar la misma a una empresa mediana o pequeña, según los recursos con los cuales se cuenta para su implementación.

El marketing en las pymes, permite: generar oportunidades, relacionadas con incremento de ventas, gracias al acercamiento que hace con sus clientes, fortalecer canal de distribución, dar a conocer mejor la organización.

En cuanto a la competencia, permite que los empresarios de las pymes, puedan conocer fortalezas y debilidades de la competencia, frente

Figura 1: Beneficios del marketing en las pymes

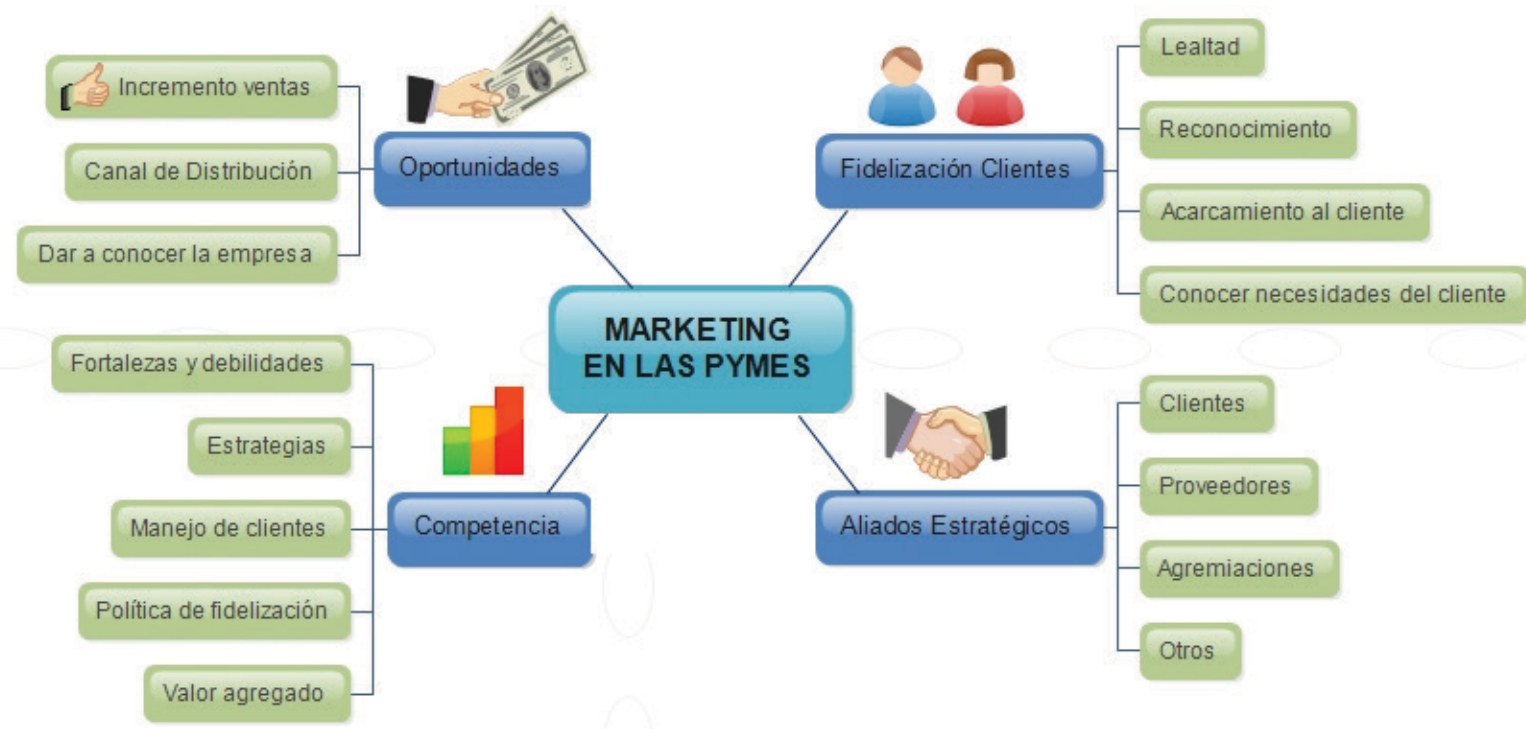

Nota: Elaboración Propia, a partir de información consultada en: Schnarch (2013). El esquema de la figura pretende resumir algunos de los beneficios que las pymes pueden tener al adoptar el Marketing como una herramienta de gestión comercial. 
a variables como: estrategias, manejo de clientes, políticas de fidelización y valor agregado.

Así mismo el marketing utilizado de manera eficiente, contribuye a la política de fidelización, por medio de actividades que permitan acercar la empresa al cliente, conocer mejor las necesidades y expectativas del cliente, generando de esta manera relaciones más estrechas con los clientes, que se pueden transformar en lealtad hacia la marca o la empresa como tal.

Por último, se puede hablar acerca de fortalecer relaciones con los aliados estratégicos como proveedores, agremiaciones, entre otros, con el propósito de estar mejor informados frente a acciones propias de la actividad comercial a la cual se dedica la empresa.

El marketing está presente en todas las acciones sociales y económicas de la cultura actual. Su importancia se hace evidente cuando se aprecia que las personas, aún sin saberlo, usan leyes de marketing en muchos actos cotidianos (Rivera \& López, 2012). En la figura 2, se podrá comprender mejor el concepto.

\section{Metodología}

Para el desarrollo de la presente publicación, se consultaron artículos científicos relacionados con el marketing y marketing en las pymes, en promedio 5 publicaciones: Kotler, Rivera \& López, Sainz y Schnarch, adicional a artículos publicados en periódicos como El Tiempo.

De igual manera se rescata la experiencia de quien escribe el artículo, en cuanto al tema de asesoría a microempresas y pymes en programas de capacitación y asesoramiento en el Sena y proyectos ejecutados en Alcaldía Local de Santafé, en años anteriores al 2014.

Partiendo del hecho de que el presente artículo pretende hacer reflexión acerca de la importancia que tiene la gestión del marketing en las pymes, se procede a indagar información que se centre en la temática, así como el aporte de la autora desde su experiencia profesional y académica.

Figura 2: Decisiones del marketing

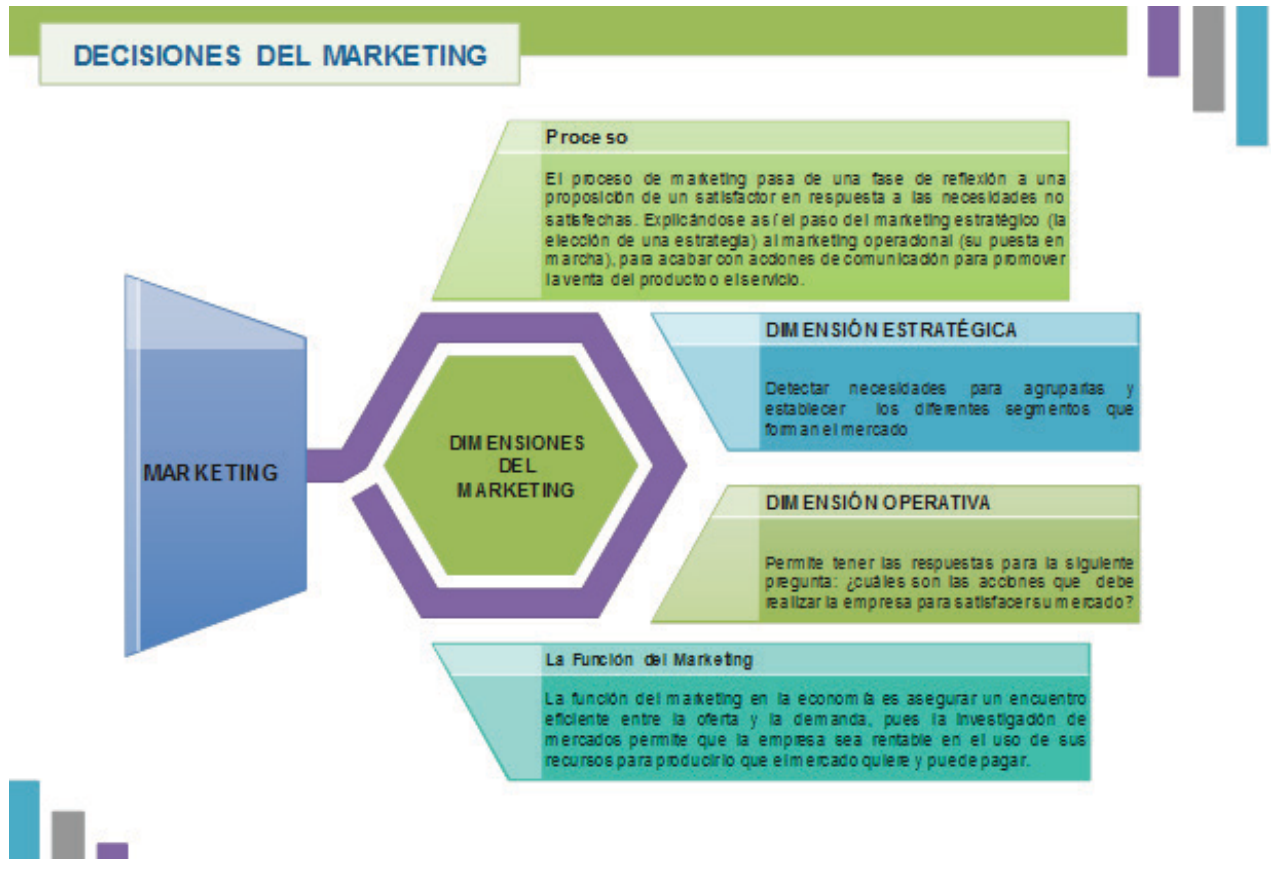

Nota: Elaboración propia, a partir de información consultada de: Rivera C. E López R. (2012). 


\section{Discusión}

Un proceso que a simple vista no parece sencillo para empresas de carácter pyme, teniendo en cuenta el nivel de inversión económica que éstas llevan a cabo para fortalecer su gestión comercial, así como su dispoción a adaptarse con facilidad a los diferentes cambios que se puedan presentar.

Precisamente el tema de actitud frente a la importancia del marketing en las pymes, se puede ver reflejado en el siguiente cuadro:

Cuadro 1: Mentalidad de Marketing vs. Mentalidad de producción.

\begin{tabular}{|l|c|c|}
\hline Problemáticas & $\begin{array}{c}\text { Mentalidad de } \\
\text { Marketing }\end{array}$ & $\begin{array}{c}\text { Mentalidad de } \\
\text { Producción }\end{array}$ \\
\hline cliente & $\begin{array}{c}\text { Las necesidades } \\
\text { del cliente } \\
\text { determinan } \\
\text { los planes } \\
\text { empresariales. }\end{array}$ & $\begin{array}{c}\text { Deben estar } \\
\text { contentos de que } \\
\text { existamos. }\end{array}$ \\
\hline Producto & $\begin{array}{c}\text { Se fabrica lo que } \\
\text { puede venderse. }\end{array}$ & $\begin{array}{c}\text { Se vende lo que se } \\
\text { fabrica }\end{array}$ \\
\hline Papel del & $\begin{array}{c}\text { Determinar las } \\
\text { necesidades del } \\
\text { cliente y cómo } \\
\text { satisfacerlas (a } \\
\text { priorí). }\end{array}$ & $\begin{array}{c}\text { Si se usa, } \\
\text { determinar la } \\
\text { reacción del cliente } \\
\text { (a posteriori) }\end{array}$ \\
\hline Publicidad & $\begin{array}{c}\text { Beneficios que } \\
\text { satisfagan las } \\
\text { necesidades }\end{array}$ & $\begin{array}{c}\text { Rasgos del producto } \\
\text { y calidad. }\end{array}$ \\
\hline
\end{tabular}

Fuente: (Sainz, 2016).

Nota: El anterior cuadro refleja, la mentalidad que se asume desde el marketing y la producción dentro de las organizaciones.

Como se puede apreciar en el anterior cuadro, hay una diferencia significativa entre la mentalidad del marketing y la mentalidad de producción; cobrando hoy en día, mayor fuerza la primera variable: mentalidad del marketing, y sobre la cual, las organizaciones deben hacer mayor énfasis, en especial las pymes, con el propósito de fortalecer su gestión comercial, conociendo mejor a sus clientes, mejorando el nivel de atención a los clientes tanto internos como externos, esto como resultado de un cliente más informado y exigente, que busca en las organizaciones el denominado valor agregado, el cual en muchas organizaciones actuales está representado en el servicio ofrecido a sus clientes, antes, durante y después de la compra.

\section{Conclusiones}

Sobre el marketing se han escrito muchos libros, investigaciones, artículos, los cuales rescatan la importancia de su aplicabilidad dentro de las organizaciones, incluso muchos de estos escritos mencionan casos exitosos en empresas grandes; pero el reto está presente en las pymes, las cuales no siempre están dispuestas a adoptar el marketing como una estrategia que permite a la organización cumplir sus metas, entre ellas la de su nivel de rentabilidad.

Siendo esta situación un reto a trabajar desde el interior de las organizaciones y a fortalecer por parte de entidades que están involucradas en el desarrollo y crecimiento de las pymes, como es el caso, por ejemplo, de la Cámara de Comercio, por medio de sus capacitaciones y asesorías; universidades con materias relacionadas con la gestión comercial, prácticas empresariales y programas de asesoramiento a unidades productivas; agremiaciones a las cuales algunas pymes están vinculadas, y otras instituciones que brinden apoyo y asesoría a empresarios de pymes. Las pymes deben comprender que el mercado está en constante cambio, que sus clientes cada vez están mejor informados, razón por la cual son más exigentes, demandando de las empresas, no sólo productos o servicios de excelente calidad, sino que esperan recibir el denominado valor agregado, sobre el cual se habla mucho hoy en día, y que en muchas ocasiones queda en sólo palabras, precisamente por falta de estructuración y desarrollo de estrategas desde la gestión del marketing. 


\section{REFERENCIAS}

El Tiempo. (13 de Febrero de 2015). Millennials, la generación que está cambiando las reglas del marketing. Recuperado de: http://blog.jwt.es/ publicidad/millennials-la-generacion-queesta-cambiando-las-reglas-del-marketing. html\#.VzSog_l97IU

Kotler, P. \& Keller, K.L. (2012). Dirección de marketing. México: Ed. Pearson.

Rivera, J. \& López, M. (2012). Dirección de marketing. Fundamentos y aplicaciones. México: Alfaomega.

Sainz, J.M. (2016). Marketing. El plan de marketing en la pyme. Madrid: Esic. Obtenido de https://books.google.es/books?id=Kh9AYSi Vf64C\&printsec $=$ copyright\&hl $=$ es\&source $=-$ gbs_pub_info_r\#v=onepage\&q\&f=false

Schnarch, A. (2013). Marketing para pymes: un enfoque para Latinoamérica. Bogotá: Alfaomega.

Semana, R. (Agosto de 1993). Revista Dinero. Recuperado de: http://www.semana.com/ nacion/articulo/historia-triunfador/20343-3 